\title{
Consumo hídrico e coeficiente de cultura da mamoneira na microrregião de Lavras, Minas Gerais ${ }^{1}$
}

\author{
Gervásio F. A. Rios², Luiz G. de Carvalho ${ }^{2}$, Flávio de C. Magina ${ }^{3}$, \\ Pedro Castro Neto ${ }^{2}$, Bruno M. Silva ${ }^{2}$ \& Antônio C. Fraga ${ }^{4}$
}

\begin{abstract}
RESUMO
Realizou-se este trabalho visando avaliar a evapotranspiração da cultura (ETc) e o coeficiente de cultivo (Kc) da cultura da mamoneira irrigada por gotejamento, sob as condições climáticas de Lavras, Minas Gerais, Brasil, no período de 24/01 a 30/08/2008. A ETc foi determinada por meio do balanço de energia, de acordo com o método da razão de Bowen, e o Kc pelos métodos e recomendações do boletim FAO 56, no período de 14/02 a 03/06/2008, correspondendo às fases I (crescimento), II (vegetativa) e III (frutificação) e para a fase IV (maturação) o Kc foi estimado por estar além do período de 03/06/2008; para isto, um experimento foi instalado no centro da área cultivada com sensores de temperatura e umidade relativa do ar, em dois níveis no dossel da cultura; de saldo de radiação (Rn) sobre o dossel da cultura e sensor de fluxo de calor no solo $(\mathrm{G})$ a 0,08 $\mathrm{m}$ da superfície do solo. Os valores médios da ETc nas fases I, II e III, foram de 2,48, 2,68 e $3,04 \mathrm{~mm} \mathrm{~d}^{-1}$, respectivamente. O Kc assumiu, nessas respectivas fases, valores de 0,27, 0,46 e 0,87 e 0,57 na fase de maturação, situando-se dentro dos limites e recomendações do boletim FAO-56.
\end{abstract}

Palavras-chave: Ricinus communis L., fluxo de calor latente, irrigação localizada, razão de Bowen, balanço de energia

\section{Water consumption and crop coefficient of castor bean in the microregion of Lavras, MG, Brazil}

\begin{abstract}
The study was carried out in order to evaluate the evapotranspiration (ETc) and crop coefficient (Kc) of castor bean by drip irrigation system, under the climatic conditions of Lavras, Minas Gerais State, Brazil, from $01 / 24$ to $08 / 30 / 2008$. The ETc was determined by the energy balance according to the Bowen ratio method and Kc by the methods and recommendations of the FAO-56 paper, from 02/14 to 06/03/2008, corresponding to phases I (growth), II (vegetative), III (fruiting) and IV (maturity). For this purpose, in the center of the experimental area, the sensors were installed to measure the temperature and relative humidity, at two levels above the crop canopy; the net radiation ( $\mathrm{Rn})$ and the soil heat flux $(\mathrm{G})$ at $0.08 \mathrm{~m}$ under the soil surface. The average ETC values in phases I, II and III were $2.48,2.68$ and $3.04 \mathrm{~mm} \mathrm{~d}^{-1}$, respectively. The respective $\mathrm{Kc}_{\mathrm{c}}$ in these phases were $0.27,0.46,0.87$ and 0.57 in maturity phase, within the limits and recommendations of the FAO-56 paper.
\end{abstract}

Key words: Ricinus communis L., latent heat flux, drip irrigation, Bowen ratio, energy balance

\footnotetext{
1 Parte da Dissertação de Mestrado do primeiro autor apresentada ao DEG/UFLA

${ }^{2}$ DEG/UFLA, Campus Universitário, Centro, C P 3037, CEP 37200-000, Lavras, MG. E-mail: gervasiorios@yahoo.com.br; Igonsaga@deg.ufla.br; pedro@oleo.ufla.br; brunoms3@yahoo.com.br

INPE, Rodovia Presidente Dutra, Km 40, C P 01, CEP 12630-000, Cachoeira Paulista, SP. E-mail: flaviomagina@uol.com.br

${ }^{4}$ DAG/UFLA, Campus Universitário, Centro, C P 3037, CEP 37200-000, Lavras, MG. E-mail: fraga@dag.ufla.br
} 


\section{INTRODUÇÃO}

A mamona (Ricinus communis L.) é uma planta da família Euphorbiaceae que, embora considerada de clima quente, adapta-se às mais variadas condições ambientais, desenvolvendo-se em climas tropicais e subtropicais (Carvalho, 2005).

Atualmente, o óleo de mamona vem sendo usado como fonte alternativa de combustível em motores de ciclo diesel, o biodiesel. Na Europa, a produção do biodiesel atende principalmente ao consumo interno. Nos Estados Unidos, leis aprovadas em Minnesota e Carolina do Norte obrigaram, desde $01 / 01 / 2002$, que todo o diesel consumido tivesse $2 \%$ de biodiesel. No Brasil foi atingida, em 2008, a meta de $2 \%$, devendo-se chegar a $5 \%$ de biodiesel incorporado ao diesel em 2013 (Paulillo et al., 2007).

Entre os produtores mundiais de mamona, em grão e óleo para exportação, o Brasil ocupa a terceira posição, depois da Índia e da China. A produção brasileira está concentrada na região Nordeste, em que o Estado da Bahia responde por mais de $90 \%$ da produção nacional, Nos estados das regiões Sul e Sudeste as produtividades médias são maiores, de $1.372 \mathrm{~kg} \mathrm{ha}^{-1}$ e $1.568 \mathrm{~kg} \mathrm{ha}^{-1}$, respectivamente. Apesar da produtividade média brasileira e mundial da mamona, em condições de sequeiro ser baixa, cerca de $600 \mathrm{~kg} \mathrm{ha}^{-1}$ (IBGE, 2009), o potencial produtivo da mamoneira é de $10.000 \mathrm{~kg} \mathrm{ha}^{-1}$ de grãos, tendo sido registrados até $8.500 \mathrm{~kg} \mathrm{ha}^{-1}$ em cultivares anãs, com o uso da irrigação (Beltrão \& Cardoso, 2006).

Neste sentido, o conhecimento e/ou determinação do consumo hídrico da cultura por meio da estimativa da evapotranspiração é fundamental, tanto do ponto de vista dos fatores de produção quanto para fins de planejamento, outorga de água, dimensionamento e manejo dos sistemas de irrigação (Allen et al., 1998; Vescove \& Turco, 2005; Barros Júnior et al., 2008; Posse et al., 2008).

Existem diversos métodos de estimativa da evapotranspiração alguns deles baseados em equações, necessitando apenas da temperatura do ar, e outros mais complexos envolvendo uma quantidade maior de elementos meteorológicos, tais como a radiação solar e a velocidade do vento. No entanto, Allen et al. (1998) e Pereira et al. (1997) ressaltam que é difícil separar a ação de cada um desses elementos visto que os mesmos agem simultaneamente. Entretanto, a evapotranspiração obtida por esses métodos corresponde à demanda atmosférica para o processo sendo, por conseguinte, uma estimativa da evapotranspiração de referência. Para a obtenção da evapotranspiração potencial da cultura, em que todas as condições do ambiente devem estar em níveis ótimos para o desenvolvimento da cultura como, por exemplo, sem deficiência de água e nutrientes ou da evapotranspiração real, caso em que a cultura pode ser de sequeiro, costuma-se aplicar outras técnicas, tais como o método do balanço de energia, aplicando-se a razão de Bowen (Perez et al., 1999; Peacock \& Hess, 2004; Silva et al., 2005;2009; Azevedo et al., 2007; 2008; Villa Nova et al., 2007; Bezerra et al., 2008; Campos et al., 2008; Souza et al., 2008; Amiro, 2009; Giambelluca et al., 2009) ou através de medições diretas em lisímetros.
Em termos práticos para que se possa conhecer a evapotranspiração de uma cultura qualquer (ETc) adota-se, normalmente, o processo de duas etapas (Pereira et al., 1997). Determina-se, inicialmente, a evapotranspiração de referência (ETo) que depois é ajustada pelo coeficiente de cultura (Kc), obtendo-se a ETc. A ETo, segundo Allen et al. (1998) é aquela que ocorre sob condições padronizadas, tendo alguns de seus fatores parametrizados e é condicionada principalmente pelos elementos meteorológicos, correspondendo à demanda atmosférica para o processo de transferência de vapor d'água para a atmosfera.

Utilizado para expressar a relação entre a ETc e a ETo, o coeficiente da cultura $(\mathrm{Kc}=\mathrm{ETc} / \mathrm{ETo})$ varia de acordo com as características da cultura e sistema de cultivo (tipo de irrigação, por exemplo), crescimento e/ou desenvolvimento da cultura, datas de plantio ou semeadura, estádio de desenvolvimento, fração de cobertura da superfície do solo pela vegetação à medida em que as plantas envelhecem e atingem a maturação e, em menor escala, com a variação dos elementos meteorológicos (Allen et al., 1998; Silva et al., 2005; Barboza Júnior et al., 2008).

O uso do Kc, variável ao longo do ciclo de cultivo, associado às estimativas da ETo tem sido apresentado e recomendado pela FAO para estimar a demanda de água das culturas em razão, dentre outras, da falta de informações específicas sobre os parâmetros que compõem o modelo de Penman-Monteith para estimativa da ETc, (Allen et al., 1998).

Apesar da expansão do cultivo e da demanda por tecnologia, são poucas as informações a respeito da necessidade hídrica da mamoneira, que possam auxiliar o manejo das irrigações, particularmente para o Estado de Minas Gerais. Neste contexto, o objetivo do trabalho foi quantificar a evapotranspiração e o coeficiente de cultura da mamoneira, sob condições de irrigação, no município de Lavras, Minas Gerais.

\section{Material e mÉtodos}

O experimento com o cultivo de mamoneira, variedade $\mathrm{AL}$ Guarany 2002, foi instalado e conduzido numa área de pesquisa medindo 0,07 ha e com topografia quase plana no Setor de Agrometeorologia e Climatologia do Departamento de Engenharia da Universidade Federal de Lavras (UFLA), em Lavras, Minas Gerais (latitude: $21^{\circ} 14^{\prime}$ S, longitude: $45^{\circ} 00^{\prime} \mathrm{W}$ e altitude: $918,84 \mathrm{~m}$ ), no período entre 24/01 a 30/08/2008. Segundo a classificação climática proposta por Köppen, o clima da região de Lavras, $\mathrm{MG}$, é Cwa, com temperatura média anual de $19,4{ }^{\circ} \mathrm{C}$, umidade relativa média anual de $76,2 \%$ e precipitação pluvial total anual de $1.530 \mathrm{~mm}$ (Dantas et al., 2007).

O preparo do solo, classificado como Latossolo Vermelho Distroférrico (EMBRAPA, 2006), consistiu de procedimentos convencionais de subsolagem, aração e gradagem e, para subsidiar os tratos culturais, foram realizadas adubação e irrigação e feitas as análises química, física e hídrica, nas camadas de 0 a 0,20 e de 0,20 a $0,40 \mathrm{~m}$.

O plantio consistiu de mudas na profundidade média de $0,15 \mathrm{~m}$, previamente formadas em viveiro, constituindo cinco fileiras de plantas espaçadas $2,40 \mathrm{~m}$ e $0,75 \mathrm{~m}$ entre plantas nas 
fileiras. A adubação de plantio foi na dosagem de $0,05 \mathrm{~kg}$ por cova (300 kg ha-1) do formulado NPK 8-28-16. Após 30 dias realizou-se a adubação de cobertura com $0,045 \mathrm{~kg}$ por planta de sulfato de amônio e os demais tratos culturais seguiram aqueles recomendados para a cultura, conforme Carvalho (2005).

O sistema de irrigação utilizado foi por gotejamento, formando uma faixa molhada (fw) de 33,3\% da área mantida praticamente constante. Tensiômetros foram instalados nas profundidades de 0,30 e 0,40 m, em dois locais da área experimental, cujas leituras foram realizadas pela manhã, utilizando-se um leitor digital de punção (tensímetro). As irrigações foram realizadas toda vez que o potencial matricial a 0,30 $\mathrm{m}$ de profundidade (3/4 da profundidade efetiva do sistema radicular, $\mathrm{z}$, considerada) atingiu o valor de $-15 \mathrm{kPa}$, considerando-se a umidade na capacidade de campo igual a $10 \mathrm{kPa}$. A lâmina de irrigação foi calculada levando-se em conta os parâmetros relativos à sua quantificação para irrigação por gotejamento, conforme procedimentos apresentados por Cabello (1996).

Para medições das variáveis necessárias ao cálculo dos componentes do balanço de energia pelo método da razão de Bowen foram instalados, em uma torre metálica com altura de 3,0 m fixada no centro da área cultivada, os seguintes sensores: um saldo radiômetro, modelo NRLITE-L25 da marca Kipp \& Zonen, na parte superior da torre avançado por um braço metálico horizontal de 1,5 m, ou seja, a 3,0 m acima do nível do solo e posicionado acima da fileira de plantas, voltado para a direção norte; dois sensores de temperatura $(\mathrm{T})$ e umidade relativa (UR) modelo HMP45C-L20 da marca Vaisala, instalados em abrigos higrotermométricos, em dois níveis, $0,5\left(Z_{1}\right)$ e $1,5 \mathrm{~m}$ $\left(Z_{2}\right)$ acima do nível do solo, fixados à torre em dois braços metálicos horizontais de $1,5 \mathrm{~m}$, voltados para o lado oposto ao do saldo radiômetro e na entrelinha de plantio; um fluxímetro da marca Campbell Scientific, Inc., modelo HFT3 instalado no solo a $0,08 \mathrm{~m}$ de profundidade, próximo ao alinhamento da projeção vertical do saldo radiômetro.

Ressalta-se que, embora a área possa ser pequena ( $0,07 \mathrm{ha})$, levando a entender que a bordadura seja insuficiente para a aplicação do método do balanço de energia, Brutsaert (1982), afirma que estudos demonstram que as relações entre 1:47 e 1:12 entre altura dos sensores e a distância limite da bordadura foram adequadas para superfícies rugosas, à semelhança da cultura da mamoneira não sendo, contudo, recomendada para superfícies heterogêneas e declivosas. Desta forma e com base na dimensão mínima acima descrita, a área atendeu à necessidade da bordadura, principalmente na direção predominante dos ventos cujo limite da área chegou a atingir 30 m de distância.

Os sensores foram conectados a um sistema automático de aquisição de dados, datalogger modelo CR-1000, da marca Campbell Scientific, Inc., no qual foram armazenados os valores médios de cada 10 minutos. Com este arranjo experimental foi possível determinar os componentes do balanço de energia, apresentados na Eq. 1. Nesta equação os componentes do balanço de energia utilizados nos processos de aquecimento do dossel das plantas $(\mathrm{P})$ e na fotossíntese $(\mathrm{F})$ não foram considerados, por representarem menos de $3 \%$ do saldo de radiação em culturas anuais (Peacock \& Hess, 2004; Villa Nova et al., 2007; Amiro, 2009).

$$
\mathrm{Rn}+\lambda \mathrm{E}+\mathrm{H}+\mathrm{G}=0
$$

A razão de Bowen é dada pela relação abaixo (Eq. 2); substituindo-a na Eq.1, chega-se à expressão que permite estimar a densidade do fluxo de calor latente (Eq. 3).

$$
\begin{gathered}
\beta=\frac{H}{\lambda E} \\
\lambda E=-\frac{R n+G}{1+\beta}
\end{gathered}
$$

em que, nessas equações, Rn é o saldo de radiação $\left(\mathrm{W} \mathrm{m}^{-2}\right) ; \lambda \mathrm{E}$ o fluxo de calor latente ( $\left.\mathrm{W} \mathrm{m}^{-2}\right)$; $\mathrm{H}$ o fluxo de calor sensível (W $\left.\mathrm{m}^{-2}\right)$ e $\mathrm{G}$ o fluxo de calor no solo $\left(\mathrm{W} \mathrm{m}^{-2}\right)$.

Convencionalmente, adotaram-se os sentidos dos fluxos como positivos, fluindo da atmosfera ou subsolo para a superfície (entrada dos fluxos no sistema solo-planta) e os fluxos negativos da superfície para a atmosfera ou subsolo (saída dos fluxos do sistema solo-planta). Os componentes Rn e $\mathrm{G}$ foram mensurados e os componentes $\lambda \mathrm{E}$ e $\mathrm{H}$, estimados com base nas equações 1 e 3 em que, assumindo-se que os coeficientes de transferência turbulenta nos processos de difusão do calor sensível e vapor d'água sejam aproximadamente iguais, isto é, $\mathrm{K}_{\mathrm{h}} \approx \mathrm{K}_{\mathrm{w}}$ e considerando-se que $\partial \mathrm{T} / \partial \mathrm{z} / \partial \mathrm{e} / \partial \mathrm{z} \approx \Delta \mathrm{T} / \Delta \mathrm{e}$, a razão de Bowen $(\beta)$ foi obtida como:

$$
\beta=\gamma \cdot \frac{\Delta \mathrm{T}}{\Delta \mathrm{e}_{\mathrm{a}}}
$$

em que, $\gamma$ é o coeficiente psicrométrico $\left(\mathrm{kPa}^{\circ} \mathrm{C}^{-1}\right) ; \Delta \mathrm{T}$ e $\Delta \mathrm{e}_{\mathrm{a}}$, as diferenças de temperatura $\left({ }^{\circ} \mathrm{C}\right)$ e pressão de vapor d'água $(\mathrm{kPa})$ entre os dois níveis $\left(Z_{1}\right.$ e $\left.Z_{2}\right)$ acima da superfície do solo, respectivamente. A pressão de vapor d'água $\left(\mathrm{e}_{\mathrm{a}}\right)$ foi estimada a partir dos dados da umidade relativa do ar (UR, em \%) e da temperatura $\left(\mathrm{T}, \mathrm{em}{ }^{\circ} \mathrm{C}\right)$, medidos nos dois níveis, mediante a definição matemática de UR, ou seja, $\left(\mathrm{e}_{\mathrm{a}} / \mathrm{e}_{\mathrm{s}}\right)_{*} 100$, em que a pressão de saturação do vapor d'água $\left(\mathrm{e}_{\mathrm{s}}\right.$, em $\left.\mathrm{kPa}\right)$ é obtida pela equação de Tetens, sendo esta e o coeficiente psicrométrico da Eq. 4 apresentados por Pereira et al. (1997). A temperatura para calcular o calor latente de evaporação, parâmetro utilizado no cálculo do coeficiente psicrométrico, correspondeu à média dos valores nos níveis de medição $\left(\mathrm{Z}_{1} \mathrm{e}\right.$ $\mathrm{Z}_{2}$ ).

Ainda no cálculo do coeficiente psicrométrico da Eq. 4 são necessários valores de pressão atmosférica sendo esses, então considerados constantes em quatro períodos $\left(\mathrm{p}_{\mathrm{i}}\right)$ do dia, $\mathrm{p}_{0}(00$ às 5 h e 50 min), $\mathrm{p}_{1}$ (06 às 11 h e 50 min ), $\mathrm{p}_{2}$ (12 às $17 \mathrm{~h}$ e $50 \mathrm{~min}$ ), $\mathrm{p}_{3}$ (18 às $23 \mathrm{~h}$ e $50 \mathrm{~min}$ ) obtidos de três medidas $\left(\mathrm{m}_{\mathrm{i}}\right)$ diárias, $\mathrm{m}_{1}$ (9h), $\mathrm{m}_{2}(15 \mathrm{~h})$ e $\mathrm{m}_{3}(21 \mathrm{~h})$ do horário local realizadas na Estação Climatológica Principal (ECP) de Lavras, localizada a $300 \mathrm{~m}$ da área experimental (pertencente ao INMET em convênio com a 
UFLA). Neste caso, a pressão atmosférica do período $\mathrm{p}_{1}$ corresponde à medida $\mathrm{m}_{1}, \mathrm{p}_{2}$ à $\mathrm{m}_{2}$ e $\mathrm{p}_{3}$ à $\mathrm{m}_{3}$, sendo $\mathrm{p}_{0}$, a pressão atmosférica diz respeito à média das medidas de $\mathrm{m}_{1}$ do dia atual e $\mathrm{m}_{3}$ do dia anterior. No cálculo da razão de Bowen, Eq. 4, valores no intervalo $[-0,9$ a - 1,1] foram eliminados, à semelhança do intervalo [-0,7 a -1,3] recomendado por Ortega-Farias et al. (1996), assim como valores maiores que 20 e menores que -20 e substituídos pela média dos valores vizinhos mais próximos.

A evapotranspiração diária da cultura (ETc) foi determinada no período entre 14/02 a 03/06/2008, pelo valor absoluto do somatório da razão entre o fluxo de calor latente de evaporação $(\lambda E)$ e o calor latente de evaporação da água $(\lambda)$, calculada a cada 10 minutos, durante as 24 h do dia, Eq. 5.

$$
\operatorname{ETc}=\left|\sum_{i=1}^{n=144}\left(\frac{\lambda E}{\lambda} \times 600\right)\right|
$$

em que:

ETc - evapotranspiração da cultura, $\mathrm{mm} \mathrm{d}^{-1}$

$\lambda$ - calor latente de evaporação da água, $\mathrm{J} \mathrm{kg}^{-1}$

600 - fator de conversão de unidades de $\lambda \mathrm{E} / \lambda\left(\mathrm{mm} \mathrm{s}^{-1}\right)$ para lâmina d'água a cada 10 min.

Para obtenção da evapotranspiração de referência (ETo) utilizou-se a metodologia proposta por Penman-Monteith, que passou por algumas parametrizações, proposta pela FAO (Food and Agricultural Organization) surgindo o método conhecido por Penman-Monteith-FAO, Eq. 6, (Allen et al., 1998).

$$
\mathrm{ETo}=\frac{0,408 \Delta(\mathrm{Rn}-\mathrm{G})+\gamma\left[\left(900 \mathrm{U}_{2}\right) /(\mathrm{T}+273)\right](\mathrm{es}-\mathrm{ea})}{\Delta+\gamma\left(1+0,34 \mathrm{U}_{2}\right)}
$$

em que $\Delta$ é a declividade da curva de pressão de saturação de vapor d'água $\left(\mathrm{kPa}^{\circ} \mathrm{C}^{-1}\right) ; \mathrm{Rn}$ é o saldo de radiação $\left(\mathrm{Mj} \mathrm{m}^{-2} \mathrm{~d}^{-1}\right)$; $\mathrm{G}$ é o fluxo de calor no solo $\left(\mathrm{Mj} \mathrm{m}^{-2} \mathrm{~d}^{-1}\right) ; \gamma$ é o coeficiente psicrométrico $\left(\mathrm{kPa}^{\circ} \mathrm{C}^{-1}\right) ; \mathrm{U}_{2}$ é a velocidade do vento média diária a $2 \mathrm{~m}$ de altura $\left(\mathrm{m} \mathrm{s}^{-1}\right)$; $\mathrm{T}$ é a temperatura média diária do ar $\left({ }^{\circ} \mathrm{C}\right)$; es é a pressão de saturação do vapor d'água média diária $(\mathrm{kPa})$; e ea é a pressão atual de vapor d'água média diária $(\mathrm{kPa})$.

O coeficiente de cultura Kc diário foi calculado segundo a relação entre a ETc e a ETo $(\mathrm{Kc}=\mathrm{ETc} / \mathrm{ETo})$ e, separadamente, foram calculados os componentes da partição de ETc, correspondentes à evaporação da água do solo (Ke:ETo) e à transpiração das plantas (KcbETo), segundo recomendações propostas por Allen et al. (1998):

$$
\begin{gathered}
\mathrm{Kc}=\mathrm{Kcb}+\mathrm{Ke} \\
\mathrm{Ke}=\mathrm{Kr} \cdot\left(\mathrm{Kc}_{\max }-\mathrm{Kcb}\right) \leq \mathrm{few} \cdot \mathrm{Kc}_{\max } \\
\text { few }=\operatorname{mín}[(1-\mathrm{fc}),(1-2 / 3 \cdot \mathrm{fc}) \cdot \mathrm{fw}]
\end{gathered}
$$

$$
\mathrm{Ke}=\left\{\begin{array}{ccc}
(1-\mathrm{few}) \cdot \mathrm{Kc}_{\max } & \text { se } & \mathrm{fc} \leq \mathrm{fw} \\
0 & \text { se } & \mathrm{fc}>\mathrm{fw}
\end{array}\right.
$$

em que, Kcb é coeficiente de transpiração da cultura; Ke é o coeficiente de evaporação da água do solo; Kr é o coeficiente de redução da evaporação; $\mathrm{Kc}_{\text {max }}$ é o coeficiente de cultura máximo; few, fração do solo molhada e exposta à atmosfera, além da projeção vertical do limite do dossel das plantas $(0,01$ - 1); fc, fração média de cobertura do solo pelo dossel da planta (0,01- 1); fw, fração média do solo molhada por irrigação e/ou precipitação (0,01-1). O Kc ${ }_{\text {max }}$ é uma função das condições meteorológicas expressas pela umidade relativa e vento e altura da planta, descrito por Allen et al. (1998).

Utilizando-se da Eq. 7 procedeu-se ao cálculo do coeficiente da cultura (Kcb) pela diferença entre Kce Ke, seguindo-se as restrições e considerações expressas na Eq. 10.

A fração de cobertura do solo pelo dossel da cultura (fc, em $\%$ ) foi determinada mediante a obtenção de valores do raio de cobertura do dossel da cultura (Rcob) como:

$$
\mathrm{fc}=\left\{\begin{array}{lll}
\frac{\pi \cdot \mathrm{Rcob}^{2}}{(\mathrm{Sp} \cdot \mathrm{Sf})} \cdot 100 & \text { se } & \text { Rcob } \leq \frac{\mathrm{Sp}}{2} \\
\frac{2 \cdot \mathrm{Rcob}}{\mathrm{Sf}} \cdot 100 & \text { se } & \text { Rcob }>\frac{\mathrm{Sp}}{2}
\end{array}\right.
$$

em que, Sp é o espaçamento entre plantas na linha de plantio (m) e Sf, o espaçamento entre linhas de plantio (m).

A curva que descreve a variação média diária de Kc foi obtida, conforme Allen et al. (1998), com os valores médios de Kc das fases I, II, III e IV sendo que o Kc da fase IV foi estimado pela média dos valores observados de Kc das fases I e III, com o intuito único e exclusivo de representação da curva inteira de Kc, tendo-se como justificava para isso a falha ocorrida no sistema de aquisição de dados nessa fase e, certamente, a constatação de que, para a grande maioria das culturas, a exemplo da mamona, os valores de Kc da fase IV ocorrem entre um valor mínimo (Kc da fase I) e um valor máximo (Kc da fase III), conforme Allen et al. (1998).

Ademais, complementando os objetivos do presente trabalho foram medidos, mensalmente, os seguintes parâmetros fenológicos: altura de planta (h), dias após o plantio (DAP) limite entre a fase inicial de crescimento, fase I, e a fase II, definido pela fração de cobertura do solo (fc) estimada em 10\%, conforme Allen et al. (1998); DAP em que mais de 50\% das plantas apresentaram o primeiro racemo (limite entre a fase de crescimento vegetativo, fase II, e a fase intermediária ou de floração e/ou frutificação, fase III); DAP em que mais de $50 \%$ dos frutos dos racemos terciários se apresentaram no estágio de maturação fisiológica (limite entre a fase III e a fase de maturação dos frutos, fase IV). Ao final do ciclo produtivo, um mês após a suspensão da irrigação, foram determinadas a produção $\left(\mathrm{kg} \mathrm{planta}^{-1}\right)$ e a produtividade da cultura $\left(\mathrm{kg} \mathrm{ha}^{-1}\right)$. 


\section{RESUlTADOS E DISCUSSÃO}

A evolução do crescimento vegetativo e das mudanças de fase da cultura por meio das variáveis altura de planta (h) e fração de cobertura do solo (fc) (Figura 1) expressa a divisão do ciclo de cultivo nas fases I, II, III e IV. A duração dessas fases foi de 36, 50, 80 e 54 dias após o plantio (DAP), respectivamente, sendo então bem mais prolongadas devido, provavelmente, à irrigação, como comentado por Allen et al. (1998).

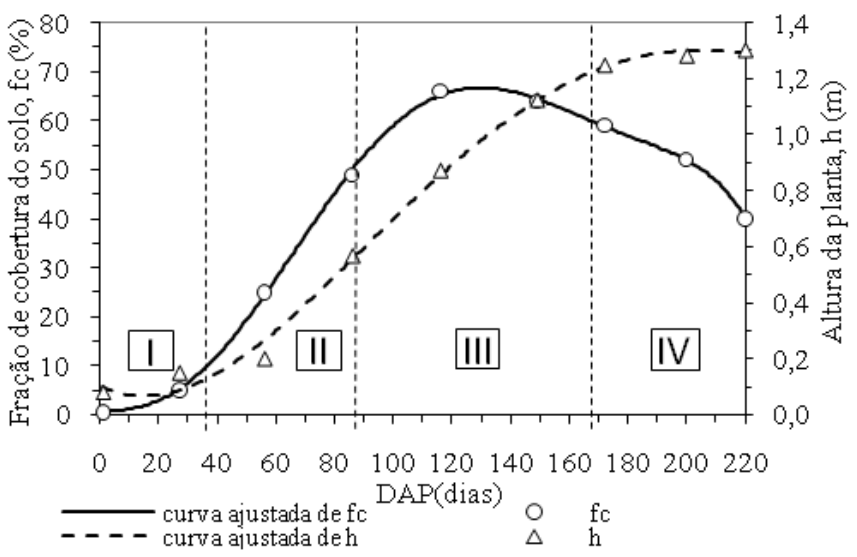

Figura 1. Fração de cobertura do solo pela cultura (fc) e altura da planta (h) ao longo das fases de cultivo (fases I, II, III, IV), de 24/01 a 30/08/2008, em dias após o plantio (DAP)

Notoriamente, como para outras culturas (Posse et al., 2008) e ainda à semelhança do que descrevem Allen et al. (1998), verifica-se que, na fase I, os valores de fc e $\mathrm{h}$ foram baixos, com taxas de crescimento lento; na fase II, a taxa de crescimento aumentou progressivamente; na fase III, a fração fc atingiu patamares mais elevados de cobertura do solo, enquanto $h$ continuou a aumentar e, na transição e durante a fase IV, a cobertura do solo ( $\mathrm{fc}$ ) reduziu consideravelmente, a taxas cada vez menores, até o mínimo de $40 \%$, enquanto a altura da planta (h) ainda aumentou, estabilizando-se e atingindo o valor máximo, 1,3 m. Contudo, pode-se observar que, de maneira geral, fc foi acompanhada de perto pelo crescimento de $h$, exceto na fase de maturação (fase IV) quando as folhas da mamona começaram a senescer e a cair sobre o solo, num comportamento semelhante ao observado por Bizinoto et al. (2010) avaliando a cultivar Al Guarany 2002 sob diferentes densidades de plantio e espaçamento.

A produção média de frutos de mamona secados ao sol foi de $0,3825 \mathrm{~kg}$ por planta e a produtividade de $2.125 \mathrm{~kg} \mathrm{ha}^{-1}$, superior à média mundial e brasileira, de cerca de $600 \mathrm{~kg} \mathrm{ha}^{-1}$, também superior as obtidas nas regiões Sul e Sudeste do País, onde as produtividades médias, foram de $1.372 \mathrm{e} 1.568 \mathrm{~kg} \mathrm{ha}^{-1}$ respectivamente (IBGE, 2009); entretanto, longe ainda do potencial produtivo da mamoneira, isto é, de $10.000 \mathrm{~kg} \mathrm{ha}^{-1}$, segundo Beltrão \& Cardoso (2006). Destaca-se que o plantio foi realizado tardiamente, incluindo o ciclo produtivo da cultura num período (janeiro a agosto) em que, notadamente, há redução das temperaturas médias e a cultura não cobriu totalmente a área útil possível de ser explorada.

Observa-se, na Figura 2 e complementando com a Tabela 1 que, em geral, as ETo e ETc foram afetadas pela nebulosidade e pelas chuvas, à semelhança do observado por Mendonça et al. (2007) e Posse et al. (2008). Os valores máximos, médios e mínimos de ETo no período de estudo, foram de 4,85, 3,47 e $1,47 \mathrm{~mm} \mathrm{~d}^{-1}$ e de $4,89,2,8$ e $0,57 \mathrm{~mm} \mathrm{~d}^{-1}$ para a ETc, respectivamente; os valores médios de ETc foram, nas fases I, II e III, de 2,48, 2,68 e $3,04 \mathrm{~mm} \mathrm{~d}^{-1}$.

Na Figura 3 se encontram os valores dos componentes evaporativos (Ke·ETo) e de transpiração das plantas (Kcb:ETo) e a curva estimada da fração de cobertura do solo (fc) durante

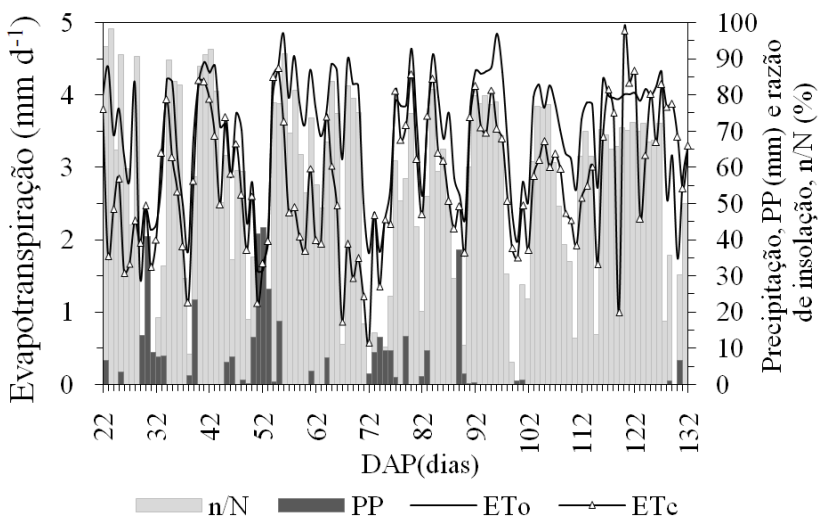

Figura 2. Evapotranspiração de referência (ETo), evapotranspiração da cultura da mamona (ETc), precipitação pluvial (PP) e razão de insolação $(\mathrm{n} / \mathrm{N})$ no período entre 22 e 132 dias após o plantio (DAP)

Tabela 1. Evapotranspiração de referência (ETo), evapotranspiração da cultura da mamona (ETc), precipitação pluvial (PP) e razão de insolação (n/N) no período entre 22 e 132 dias após o plantio (DAP) em médias quinquenais

\begin{tabular}{|c|c|c|c|c|c|c|c|c|c|}
\hline \multirow{2}{*}{$\begin{array}{l}\text { DAP } \\
\text { dias }\end{array}$} & \multirow{2}{*}{$\begin{array}{l}\mathrm{n} / \mathrm{N} \\
\mathrm{adm}\end{array}$} & PP & ETo & ETC & \multirow{2}{*}{$\begin{array}{l}\text { DAP } \\
\text { dias }\end{array}$} & \multirow{2}{*}{$\begin{array}{l}\mathrm{n} / \mathrm{N} \\
\mathrm{adm}\end{array}$} & PP & ETo & ETc \\
\hline & & & $\mathrm{mm}$ & & & & \multicolumn{3}{|c|}{$\mathrm{mm}$} \\
\hline $22-26$ & 81,5 & 2,0 & 3,8 & 2,5 & $77-81$ & 57,6 & 3,0 & 4,0 & 3,7 \\
\hline $27-31$ & 37,1 & 12,6 & 2,6 & 2,0 & $82-86$ & 55,5 & 2,3 & 3,6 & 3,3 \\
\hline $32-36$ & 61,5 & 3,1 & 3,3 & 3,0 & $87-91$ & 34,2 & 8,0 & 3,1 & 2,5 \\
\hline $37-41$ & 54,8 & 5,2 & 3,3 & 2,9 & $92-96$ & 78,5 & 0,1 & 4,4 & 3,8 \\
\hline $42-46$ & 64,3 & 2,7 & 3,8 & 3,3 & $97-101$ & 26,6 & 0,4 & 3,0 & 2,4 \\
\hline $47-51$ & 34,2 & 11,2 & 2,8 & 2,3 & $102-106$ & 63,2 & 0,0 & 3,7 & 2,8 \\
\hline $52-56$ & 51,8 & 17,6 & 3,4 & 3,2 & $107-111$ & 39,2 & 0,0 & 3,3 & 2,5 \\
\hline $57-61$ & 68,2 & 0,7 & 4,1 & 2,3 & $112-116$ & 56,0 & 0,0 & 3,7 & 2,7 \\
\hline $62-66$ & 65,9 & 1,5 & 4,0 & 2,6 & $117-121$ & 68,1 & 0,0 & 4,0 & 3,6 \\
\hline $67-71$ & 52,9 & 0,0 & 3,7 & 1,5 & $122-126$ & 69,8 & 0,0 & 4,0 & 3,4 \\
\hline $72-76$ & 9,7 & 8,6 & 2,2 & 1,8 & $127-132$ & 34,2 & 1,2 & 3,0 & 3,6 \\
\hline
\end{tabular}


o período de estudo, distinguindo-se os 67 DAP como sendo o dia em que fc ultrapassou a fração de solo molhada pela irrigação na linha de plantio (fw) resultando no componente Ke:ETo praticamente nulo. Esta condição foi ainda mais eminente após os $70 \mathrm{DAP}$, período correspondente à diminuição das chuvas (Figura 2), no qual a ETc esteve mais restrita ao componente de transpiração, razão por que se verifica, na Figura 3, como já descrito por diversos autores (Pereira et al., 1997; Allen et al., 1998; Mendonça et al., 2007; Posse et al., 2008), que o componente transpirativo (KcbETo) acompanha, em termos médios, a curva de fc em todo o período analisado e o componente evaporativo (KeETo) foi maior no período de maior concentração das chuvas e exposição do solo à radiação solar.

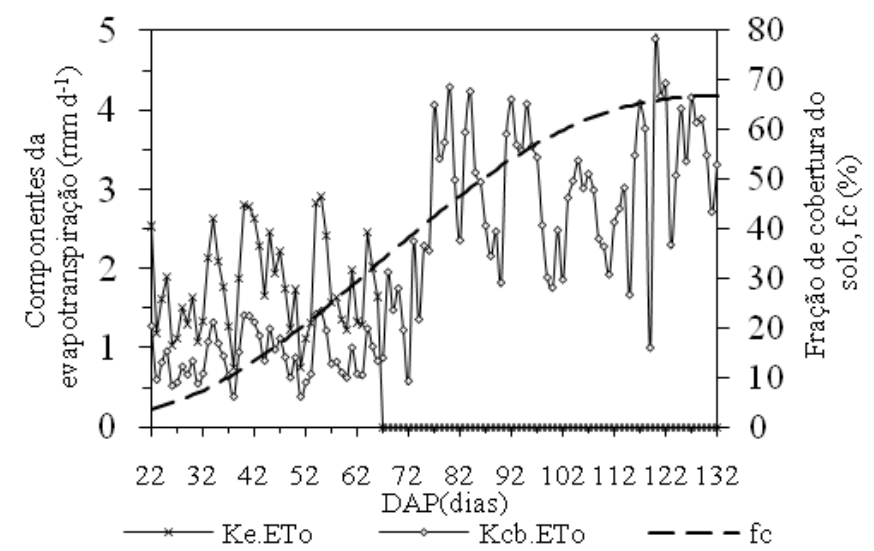

Figura 3. Curvas dos componentes de evaporação do solo (Ke·ETo) e transpiração da cultura da mamona (Kcb-ETo) e curva da fração de cobertura do solo pela cultura (fc) no período entre 22 e 132 dias após o plantio (DAP)

Observa-se, na Figura 4, o comportamento da curva de Kc nas quatro fases do ciclo, dos valores de Kc observados e da curva de fc, verificando-se que esses valores de Kc acompanham, em termos médios, a curva de fc em todo o período analisado, com ajuste da curva de segundo grau apresentando coeficiente de determinação $\left(r^{2}\right)$ de 0,635 , ou seja, entre Kc e fc.

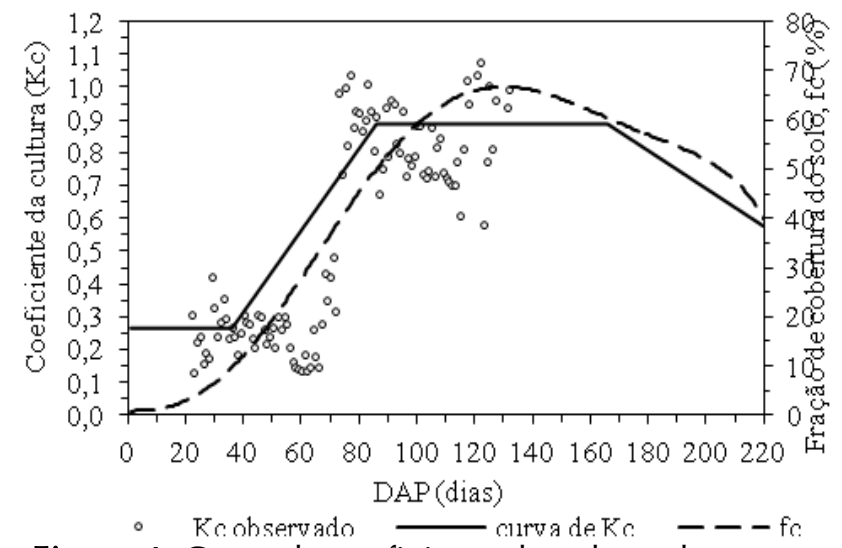

Figura 4. Curva do coeficiente de cultura da mamona Kc e valores de Kc observados e curva da fração de cobertura do solo pela cultura (fc) entre 24/01 a 30/08/ 2008, em dias após o plantio (DAP)
Salienta-se a falha no sistema de aquisição de dados a partir dos 130 DAP. A fase III se estendeu de 86 aos 166 DAP; apesar disto e se considerando que a quantidade de valores de $\mathrm{Kc}$ observados nesta fase atingiu cerca de $57 \%$ do período, ou seja, 46 dias (86 aos 132 DAP) e, ainda, que com o pico da curva de fc nesta fase se pode inferir que o valor médio encontrado se estende até o final do período correspondente. $\mathrm{O} \mathrm{Kc}$ na fase IV foi estimado pela média dos valores observados de Kc das fases I e III, com o intuito único e exclusivo de representação da curva completa de Kc pois, conforme descrevem Allen et al. (1998), para a grande maioria das culturas, os valores de $\mathrm{Kc}$ da fase IV ocorrem entre um valor mínimo (Kc da fase I) e um valor máximo (Kc da fase III).

Os valores médios de Kc nas fases I, II, III e IV foram, respectivamente, de 0,27, 0,46, 0,87 e 0,57. Por sua vez, Allen et al. (1998) apresentam, em tabelas, valores médios padronizados, ou seja, em condições climáticas médias de umidade relativa mínima média do ar igual a $45 \%$, velocidade média do vento a $2 \mathrm{~m}$ de altura de $2 \mathrm{~m} \mathrm{~s}^{-1}$ e altura máxima média da cultura de $0,3 \mathrm{~m}$, de $\mathrm{Kc}$ iguais a $0,35,1,15$ e 0,55 para a mesma cultura. Ajustando esses valores $(0,35,1,15$ e 0,55$)$, de acordo com os critérios também apresentados por Allen et al. (1998) para as condições meteorológicas médias do período experimental do presente trabalho (umidade relativa mínima, $50 \%$; vento a $2 \mathrm{~m}$ de altura, $1,5 \mathrm{~m} \mathrm{~s}^{-1}$ e altura da cultura, $1,3 \mathrm{~m}$ ), se encontraram os Kc iguais a $0,35,1,12$ e 0,52, respectivamente, das fases I, III e IV. Evidentemente, como discutem Allen et al. (1998), Silva et al. (2005) e Barboza Júnior et al. (2008), tais valores diferem dos Kc determinados no presente estudo $(0,27,0,46,0,87$ e 0,57$)$, podendo-se atribuílos às condições experimentais e, sobretudo, ao sistema de irrigação localizada, contribuindo para um consumo menor de água, levando a menores valores de Kc.

Com base nesses valores de $\mathrm{Kc}$ ajustados $(0,35,1,15$ e 0,55), são obtidos os valores médios de Kcb também ajustados para as fases I, III e IV, de acordo com os critérios propostos por Allen et al. (1998). Para tanto, basta subtrair, em 0,2, 0,1 e 0,1, os Kc ajustados das fases I, III e IV, respectivamente, obtendo-se, portanto, os Kcb ajustados e ou recomendados $(0,15,1,02 \mathrm{e}$ 0,42 ) na mesma ordem. Esses últimos, segundo os autores, apesar de serem mais representativos quando obtidos in loco, não devem diferir em mais de 0,2 dos valores de Kcb recomendados. Desta forma e se aplicando os mesmos critérios para os Kc do presente trabalho $(0,27,0,87$ e 0,57$)$, se encontraram os valores de 0,07, 0,77 e 0,47. Verifica-se que esses valores médios de Kcb das fases I e IV determinados, estão dentro da tolerância, à exceção do Kc da fase III.

Segundo Allen et. al. (1998), nas fases III e IV há uma aproximação maior da curva de Kc à curva de Kcb que, por definição, representa a curva base (por isso o nome dado de coeficiente de cultura basal), pois o desenvolvimento do dossel vai sombreando progressivamente o solo, reduzindo a componente evaporativa da evapotranspiração; para a grande maioria das culturas esta aproximação nas fases III e IV é esperada, a depender principalmente da cultura, das práticas culturais, fração de cobertura do solo, ocorrência de chuvas, do tipo e frequência de irrigação. 


\section{CONCLUSÕES}

1. Os valores médios da evapotranspiração da cultura da mamona (ETc) foram de 2,48, 2,68 e 3,04 $\mathrm{mm} \mathrm{d}^{-1}$ para as fases inicial, de crescimento e intermediária, respectivamente.

2. Os respectivos valores de coeficiente da cultura $(\mathrm{Kc})$ nas fases inicial, crescimento e intermediária, são 0,27, 0,46 e 0,87 e ainda 0,57 para o final da cultura.

\section{Agradecimentos}

Ao Departamento de Engenharia da UFLA, pela estrutura e transferência de conhecimentos; À CAPES, pela concessão de bolsa ao primeiro autor; À FAPEMIG, pelo financiamento do projeto e à FINEP, pela disponibilidade de recursos.

\section{LITERATURA CITADA}

Allen, R. G.; Pereira, L. S.; Raes, D.; Smith, M. Crop evapotranspiration: Guidelines for computing crop water requirements. Rome: FAO, 1998. 297p. Irrigation and Drainage Paper: 56.

Amiro, B. Measuring boreal forest evapotranspiration using the energy balance residual. Journal of Hydrology, v.366, p.112-118, 2009.

Azevedo, P. V. de; Soares, J. M.; Silva, V. de P. R. da; Silva, B. B. da; Nascimento, T. Evapotranspiration of superior grapevines under intermittent irrigation. Agricultural Water Management, v.95, p.301-308, 2008.

Azevedo, P. V. de; Souza, C. B. de; Silva, B. B. da; Silva, V. de P. R. da. Water requirements of the pineapple crop grown in tropical environmentals, Brazil. Agricultural Water Management, v.88, p.201-208, 2007.

Barboza Júnior, C. R. A.; Folegatti, M. V. ; Rocha, F. J.; Atarassi, R. T. Coeficiente de cultura da lima-ácida tahiti no outonoinverno determinado por lisimetria de pesagem em Piracicaba - SP. Engenharia Agrícola, v.28, p.1-10, 2008.

Barros Júnior, G.; Guerra, H. O. C.; Cavalcanti, M. L. F.; Lacerda, R. D. de. Consumo de água e eficiência do uso para duas cultivares de mamona submetidas a estresse hídrico. Revista Brasileira de Engenharia Agrícola e Ambiental, v.12, p.350355, 2008.

Beltrão, N. E. de M.; Cardoso, G. D. Informações sobre o sistema de produção utilizados na ricinocultura na região nordeste, em especial o semi-árido e outros aspectos ligados a sua cadeia. Campina Grande: Embrapa Algodão, 2006. 6p. Comunicado Técnico, 213.

Bezerra, B. G.; Silva, B. B. da; Ferreira, N. J. Estimativa da evapotranspiração real diária utilizando-se imagens digitais TM - Landsat 5. Revista Brasileira de Meteorologia, v.23, p.305-317, 2008.

Bizinoto, T. K. M. C.; Oliveira, E. G. de; Martins, S. B.; Souza, S. A. de; Gotardo, M. Cultivo da mamoneira influenciada por diferentes populações de plantas. Bragantia, v.69, p.367370, 2010.
Brutsaert, W. Evapotranspiration into the atmosphere: theory, history and applications. Dordrecht: D. Reidel, 1982. 229p.

Cabello, F. P. Riegos localizados de alta frecuencia. Madrid: Ediciones Mundi-Prensa, 1996. 511p.

Campos, J. H. B. da C.; Silva, V. P. R. da; Azevedo, P. V. de; Borges, C. J. R.; Soares, J. M.; Moura, M. S. B. de; Silva, B. B. da. Evapotranspiração e produtividade da mangueira sob diferents tratamentos de irrigação. Revista Brasileira de Engenharia Agrícola e Ambiental, v.12, p.150156, 2008.

Carvalho, B. C. L. Manual do cultivo da mamona. Salvador: EBDA, 2005. 65p.

Dantas, A. A. A.; Carvalho, L. G. de; Ferreira, E. Classificação e tendência climática em Lavras, MG. Ciência e Agrotecnologia, Lavras, v.31, p.1862-1866, 2007.

EMBRAPA - Empresa Brasileira de Pesquisa Agropecuária. Sistema Brasileiro de Classificação de Solos. 2.ed. Rio de Janeiro: Embrapa Solos, 2006. 306p.

Giambelluca, T. W.; Martin, R. E.; Asner, G. P.; Huang, M.; Mudd, R. G.; Nullet, M. A.; DeLay, J. K.; Foote, D. Evapotranspiration and energy balance of native wet montane cloud forest in Hawai'i. Agricultural and Forest Meteorology, v.149, p.230243, 2009.

IBGE - Instituto Brasileiro de Geografia e Estatística. Sidra: banco de dados agregado. <http://www.sidra.ibge.gov.br/ bda/tabela/listabl.asp?c=99\&z=p\&o=18.>. 05 Set. 2009.

Mendonça, J. C.; Sousa E. F. de; Bernardo, S.; Sugawara, T. M.; Peçanha, A. L.; Gottardo, R. D. R. Determinação do coeficiente cultural (Kc) do feijoeiro (Phaseolus vulgaris L.), em Campos dos Goytacazes, RJ. Revista Brasileira de Engenharia Agrícola e Ambiental, v.11, p.471-475, 2007.

Ortega-Farias, S. O.; Cuenca, R. H.; Ek, M. Daytime variation of sensible heat flux estimated by the bula aerodynamic method over a grass canopy. Agricultural and Forest Meteorology, v.81, p.131-143, 1996.

Paulillo, L. F.; Vian, C. E. de F. ; Shikida, P. F. A.; Mello, F. T. de. Álcool combustível e biodiesel no Brasil: Quo vadis? Revista de Economia e Sociologia Rural, v.45, p.531-565, 2007.

Peacock, C. E.; Hess, T. M. Estimating evapotranspiration from a reed bed using the Bowen ratio energy balance method. Hydrological Processes, v.18, p.247-260, 2004.

Pereira, A. R.; Villa Nova, N. A.; Sediyama. G. C. Evapo(transpi)ração. Piracicaba: FEALQ, 1997. 183p.

Perez, P. J.; Castellvi, F.; Ibañez, M.; Rosell, J. I. Assessment of reliability of Bowen ratio method for partitioning fluxes. Agricultural and Forest Meteorology, v.97, p.141-150, 1999.

Posse, R. P.; Bernardo, S.; Sousa, E. F. de; Gottardo, R. D. Evapotranspiração e coeficiente da cultura do mamoeiro. Engenharia Agrícola, v.28, p.681-690, 2008.

Silva, L. D. B. da; Folegatti, M. V.; Nova, N. A. V.. Evapotranspiração do capim Tanzânia obtida pelo método de razão de Bowen e lisímetro de pesagem. Engenharia Agrícola, v.25, p.705-712, 2005. 
Silva, V. de P. R. da; Campos, J. H. B. da C.; Azevedo, P. V. de. Water use efficiency and evapotranspiration of mango orchard grown in northeastern of Brazil. Scientia Horticulturae, v.120, p.467-472, 2009.

Souza, C. B. de; Silva, B. B. da; Azevedo, P. V. de; Silva, V. de P. R. da. Fluxos de energia e desenvolvimento da cultura do abacaxizeiro. Revista Brasileira de Engenharia Agrícola e Ambiental, v.12, p.400-407, 2008.
Vescove, H. V.; Turco, J. E. P. Comparação de diferentes métodos de estimativa da evapotranspiração de referência para a região de Araraquara SP. Engenharia Agrícola, v.25, p.713$721,2005$.

Villa Nova, N. A.; Pereira, A. B.;. Shock, C. C. Estimation of reference evapotranspiration by an energy balance approach. Biosystems Engineering, v.96, p.605-615, 2007. 